\title{
Mechanical interventricular dependency supports hemodynamics in tako-tsubo cardiomyopathy
}

\author{
Juan Lei ${ }^{1,2 \#}$, Zhongxia Sun ${ }^{1,3 \#}$, Lingchun Lyu ${ }^{1,4}$, Randall G. Green ${ }^{5}$, Ernest Scalzetti ${ }^{6}$, David Feiglin ${ }^{6}$, \\ Jingfeng Wang ${ }^{2}$, Kan Liu ${ }^{1}$ \\ ${ }^{1}$ Division of Cardiology, State University of New York, Upstate Medical University Hospital, Syracuse, NY, USA; ${ }^{2}$ Department of Cardiology, Sun \\ Yat-sen Memorial Hospital, Sun Yat-sen University, Guangzhou 510120, China; ${ }^{3}$ Department of Ultrasonography, the First Affiliated Hospital, \\ ${ }^{4}$ Department of Cardiology, Lishui Hospital, Zhejiang University, Hangzhou 310003, China; ${ }^{5}$ Division of Cardiac Surgery, Department of Surgery, \\ ${ }^{6}$ Department of Radiology, State University of New York, Upstate Medical University Hospital, Syracuse, NY, USA \\ Contributions: (I) Conception and design: J Lei, Z Sun, J Wang, K Liu; (II) Administrative support: None; (III) Provision of study materials or \\ patients: E Scalzetti, D Feiglin; (IV) Collection and assembly of data: J Lei, Z Sun; (V) Data analysis and interpretation: J Lei, Z Sun, RG Green; (VI) \\ Manuscript writing: All authors; (VII) Final approval of manuscript: All authors. \\ "These authors contributed equally to this work. \\ Correspondence to: Kan Liu, MD, PhD. Division of Cardiology, State University of New York, Upstate Medical University, 90 presidential plaza, \\ Syracuse, NY 13202, USA. Email: liuk@upstate.edu.
}

Background: Although morphological abnormalities of the heart appear to be remarkable, most patients with tako-tsubo cardiomyopathy (TTC) remain clinically stable. We investigate real time changes in the left ventricular (LV) and right ventricular (RV) mechanics and function to explore the mechanism to preserve hemodynamics.

Methods: With deformation and Doppler echocardiography, we evaluated myocardial mechanics and ventricular function/hemodynamics simultaneously in 103 consecutive TTC patients admitted from 01/01/2008 through 12/31/2015. The coronary angiography and left ventriculography were performed to rule out culprit coronary artery stenosis (CAS). We included 66 patients in a control group with matched age, sex, and risk factors for coronary artery disease (CAD), and 41 patients in a group of myocardial infarction induced cardiogenic shock, who required circulatory supporting devices to maintain hemodynamic stability.

Results: Although systolic myocardial strain in most of the LV segments was significantly impaired, 4 basal LV segments remained functionally active during acute stage of TTC. The impairment in the myocardial strain of the RV apex could extend to the middle segments, but basal RV systolic strain was also preserved. Despites comparable apical to basal strain gradients, LV and RV displayed discrepant functional/ hemodynamic status. In contrast to LV, RV functional/hemodynamic parameters appeared to be hyperdynamic. This unique RV strain pattern remained unchanged in patients with atypical (mid-LV cavity) TTC. In 41 patients with myocardial infarction induced cardiogenic shock, RV exhibited comparable mechanic and functional features with those in TTC patients.

Conclusions: The identified LV and RV mechanic changes appear to support interventricular hemodynamic dependence during TTC, which may represent a universal rescue mechanism in a jeopardized or injured heart.

Keywords: Tako-tsubo cardiomyopathy (TTC); hemodynamics; deformation; strain; right ventricle

Submitted Jan 31, 2018. Accepted for publication Apr 18, 2018.

doi: $10.21037 /$ jtd.2018.04.143

View this article at: http://dx.doi.org/10.21037/jtd.2018.04.143 


\section{Introduction}

Applying more real time cardiac imaging in patients with acute coronary syndrome, we begin to realize that tako-tsubo cardiomyopathy (TTC) occurs more often than we originally believed $(1,2)$. Despite abrupt cardiac morphological changes and apparent ventricular "dysfunction" in anatomic images, most TTC patients are clinically asymptomatic. Meanwhile, acute congestive heart failure with hemodynamic instability can sometimes occur unpredictably, resulting in adverse sequelae (2-4). Understanding the unique pathophysiology of TTC helps direct appropriate management, avoid harmful pharmaceutical/device therapies, and prevent adverse events/outcome.

In this study, we aimed to characterize real time changes of the left ventricular (LV) and right ventricular (RV) mechanics and functions with integrated deformation and Doppler echocardiography, to explore one possible mechanism contributing to hemodynamic maintenance during TTC.

\section{Methods}

\section{Patient population}

We included 3 patient groups in this study: (I) TTC patients; (II) patients with AMI induced cardiogenic shock (CS); (III) control patients without structural/functional cardiac abnormality (Figure 1 and Table 1).

We retrospectively completed a systematic search of the computerized database Epic Electronic Medical Record, including the data from echocardiography and cardiac catheterization laboratories of State University of New York (SUNY) Upstate Medical University, and identified consecutive TTC patients from 01/01/2008 through $12 / 31 / 2015$. The inclusion criteria for patients diagnosed as TTC follows the updated diagnostic criteria $(5,6)$ : (I) characteristic left ventricular (LV) segment wall motion abnormalities, including apical ballooning and mid-ventricular akinesis/dyskinesis on the initial left ventriculography or echocardiography; (II) ST-segment or T-wave abnormalities on electrocardiography (ECG) and increases in blood concentrations of cardiac troponin T/creatine kinase MB; (III) no culprit coronary artery stenosis (CAS) revealed by coronary angiogram (CAG); (IV) if there were contraindications for an immediate cardiac catheterization, the diagnosis would be made based on follow up echocardiograms documenting complete normalization of cardiac systolic function/segmental wall motion without cardiovascular revascularization, and
CAG or stress echocardiography/cardiovascular single-photon emission computed tomography to rule out the existence of culprit CAS.

Patients with matched age, sex, and risk factors for coronary artery disease (CAD) from of SUNY Upstate Medical University evaluated/treated during 01/01/2013 through 12/31/2015 were chosen as controls. The controls patients were initially referred for echocardiography for chest pain, dyspnea, palpitation, or syncope, or for new onset abnormal ECG changes, in the absence of any prior history of structural heart disease, and subsequent workup ruled out any cardiac structural/function (systolic) abnormalities, including significant heart valve diseases. The "absence" of LV and RV structural heart disease" in the echocardiographic exam was defined according to published American Society of Echocardiography guidelines $(7,8)$. An age- and gender-matched comparator group was selected according to published comparability principles (9).

From consecutive patients with CS (defined as systolic blood pressure $<90 \mathrm{mmHg}$ with signs of tissue hypoperfusion requiring inotropic agents and/or fluid therapy) (10) from 01/01/2013 through $12 / 31 / 2015$, we included those who had significant LV systolic dysfunction secondary to new onset CAG-proven AMI, and required a circulatory supporting device (intra-aortic balloon pump, percutaneous LV assist device-Impella 2.5, or venoarterial extracorporeal membrane oxygenation) to maintain hemodynamic stability. The finding of subtotal or total occlusion of coronary arteries was diagnosed as the culprit lesion on CAG and related wall motion abnormalities were revealed by either ventriculography or echocardiography. Patients with ongoing arrhythmia and/or pre-existing significant valvular heart disease or pulmonary arterial hypertension were excluded.

Clinical variables included demographic data and the presence of the risk factors for $\mathrm{CAD}$, including diabetes, hypertension, hyperlipidemia and smoking. Laboratory variables included peak serum levels of troponin $T$, and creatine kinase MB. A standard 12-lead surface ECG was performed in all patients.

All of the research proposals/protocols have been reviewed and approved by the institutional research board of the Human Subjects Committee of SUNY Upstate Medical University.

\section{Cardiac catheterization with CAG}

Cardiac catheterizations with selective CAG were 


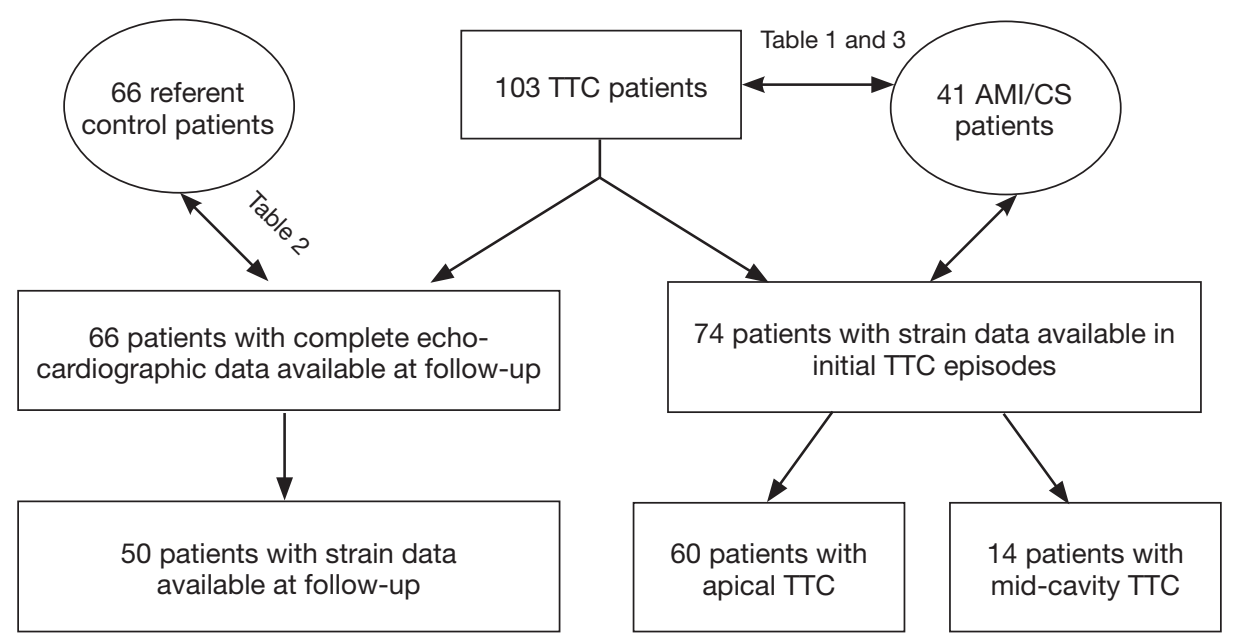

Figure 1 Flow diagram illustrating patient selection for this study. Patients with TTC and AMI/CS were culled from Epic electronic medical record, including data from the catheterization and echocardiography laboratory database. Follow-up echocardiographic studies were completed in TTC patients at their outpatient visits in the cardiology clinic. Controls were chosen with matched age, sex, and risk factors for coronary artery disease, who had initial clinical symptoms of chest pain or dyspnea, but subsequent workup ruled out any cardiac structural/functional abnormalities. TTC, tako-tsubo cardiomyopathy; AMI, acute myocardial infarction; CS, cardiogenic shock.

Table 1 Clinical characteristics of control, TTC and CS patients

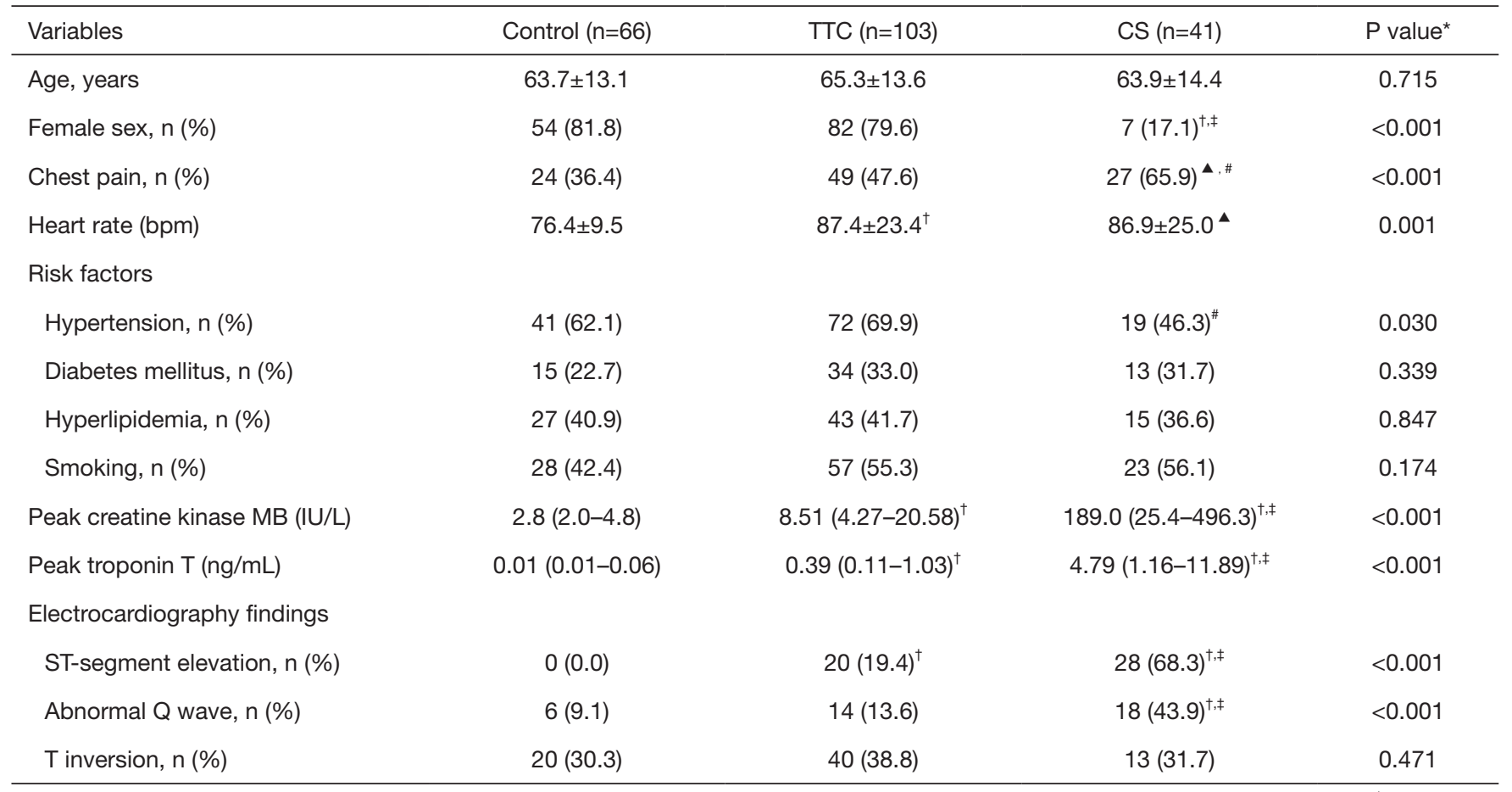

Values are presented as mean \pm standard deviations, $\mathrm{n}(\%)$ or median (interquartile range). *, $\mathrm{P}$ value from one way ANOVA; ${ }^{\star}, \mathrm{P}<0.05$ compared with control; ${ }^{\dagger}, \mathrm{P}<0.01$ compared with control; *, $\mathrm{P}<0.05$ compared with TTC; ${ }^{\ddagger}, \mathrm{P}<0.01$ compared with TTC; CS, cardiogenic shock; TTC, tako-tsubo cardiomyopathy. 
performed with standard techniques. Left ventriculography was performed with a pigtail catheter. Results were recorded in both standard reports and digital imaging. LV pressures aortic systolic and diastolic pressure were recorded, angiographic $\mathrm{LV}$ volumes was computed with standard methods (11).

\section{Two dimensional (2D) and Doppler echocardiography}

Transthoracic echocardiography (TTE) was performed in all patients within 24 hours of admissions. Followup echocardiographic studies were completed within 8-12 weeks in 66 TTC patients at their follow-ups in our cardiology faculty clinic. TEE was performed with standard techniques using GE Vivid E9 machine (GE Healthcare, Vingmed Ultrasound A/S, Norway) with a $3.5-\mathrm{MHz}$ phasedarray transducer was used for standard comprehensive twodimensional (2D) Doppler echocardiography.

\section{LV/RV function and bemodynamic assessment}

The LV ejection fraction (LVEF) was calculated by biplane Simpson's method from apical 4- and 2-chamber views. RV ejection fraction (RVEF) was calculated by the single plane Simpson's method from apical 4-chamber view. Tei index, aortic jet time, RV outflow tract (RVOT) diameter, velocity time integral (VTI) across the RVOT, RV fractional area change (FAC) and tricuspid annular plane systolic excursion (TAPSE) were performed following the updated guidelines of the Society of Echocardiography $(7,8)$. Both blood flow velocity across the mitral valve (E) and mitral annulus (myocardial) velocity (e') were measured during early diastole. Tricuspid regurgitant (TR) dP/dt was obtained from the continuous wave Doppler envelope of the TR jet from 1 to $2 \mathrm{~m} / \mathrm{s}$, according to the simplified Bernoulli equation $(12,13)$.

\section{Ventricular speckle tracking longitudinal strain analysis}

2D grayscale images were acquired in the standard apical views. Only images with frame rates $>40$ frames/sec were selected for reliable analysis. All images were stored digitally for subsequent offline analysis. Speckle tracking analysis automatically tracked myocardial motion throughout the cardiac cycle and allowed a rapid generation of regional myocardial strain curves. We traced the endocardial border at end of ventricular systole and adjusted the width of the ventricular walls to include the entire myocardium. Built- in software automatically accepted segments with good tracking quality, and rejected segments with poor tracking quality. Finally, an automated function imaging based on speckle tracking analysis integrated the quantitative data of ventricular peak systolic longitudinal strain into a standard 18-segment model with a "bulls-eye" figure, helping detect both regional and global ventricular dysfunction. The 18 LV myocardial segments were grouped as basal, middle and apical segments (with 6 segments in each group) for the statistical analysis, and the strain value of each group was calculated by averaging the peak systolic longitudinal strain of the 6 segments $(14,15)$. Endocardial borders of the RV in apical 4 chamber view were manually traced from the septal-atrioventricular annular hinge point to apical septum and to RV lateral wall at the lateral atrio-ventricular annular hinge point. ECHOPAC speckle tracking software was applied to RV to produce six segmental (basal septum, mid septum, apical septum, basal RV, mid RV and apical RV) peak strain curves and time to peak intervals. The interventricular septum (basal septum, mid septum, apical septum) was partially attributed to LV. Therefore, the peak systolic longitudinal strain in our study was assessed in the 3 lateral RV segments, defined as the lateral basal, lateral mid, and lateral apical segments (16-18).

\section{Statistical analysis}

Quantitative variables showing a normal distribution, data are expressed as mean \pm standard deviations, and compared using Student $t$-test or one way ANOVA. Quantitative variables showing a non-normal distribution, data are expressed as median (interquartile range) and compared using Mann-Whitney U test or KruskalWallis test. Categorical data are presented as number and percentage and compared using $\chi^{2}$ test or Fisher exact test. Statistical significance was established at 2 -tailed $\mathrm{P}<0.05$. The Bonferroni post hoc test for between-group pairwise comparisons was done with significant differences evaluated at $\mathrm{P}<0.01$. All statistical analyses were performed with SPSS Statistics for Windows, version 22 (IBM Corporation, Armonk, New York, USA).

\section{Results}

\section{Demographic and laboratory characteristics}

Table 1 summarized the demographic and selected laboratory data obtained in control, TTC and AMI/CS 
Table 2 Functional/hemodynamic parameters in controls, TTC patients and TTC patients after recovery

\begin{tabular}{|c|c|c|c|c|}
\hline Variables & Control $(n=66)$ & TTC $(n=66)$ & TTC after recovery $(n=66)$ & $P$ value \\
\hline LVEF (\%) & $60.2 \pm 7.3$ & $38.5 \pm 7.4^{\dagger}$ & $55.5 \pm 7.4^{\dagger, \neq}$ & $<0.001$ \\
\hline E/A ratio & $1.03(0.81-1.24)$ & $0.97(0.69-1.37)$ & $0.95(0.75-1.42)$ & 0.575 \\
\hline Tei index & $0.37 \pm 0.18$ & $0.70 \pm 0.29^{\dagger}$ & $0.44 \pm 0.17^{\ddagger}$ & $<0.001$ \\
\hline $\mathrm{b}(\mathrm{ms})$ & $311.4 \pm 41.9$ & $237.5 \pm 49.5^{\dagger}$ & $286.2 \pm 46.0^{\dagger, \ddagger}$ & $<0.001$ \\
\hline E/e' ratio & $9.0(6.9-11.2)$ & $11.8(9.5-16.2)^{\dagger}$ & $9.6(7.5-11.9)$ & $<0.001$ \\
\hline \multicolumn{5}{|l|}{ Right ventricular } \\
\hline RVEDV (mL) & $32.5(23.3-40.8)$ & $24.5(18.0-33.8)^{\dagger}$ & $31.0(24.0-40.8)$ & 0.009 \\
\hline FAC (\%) & $42.8 \pm 5.1$ & $48.1 \pm 9.3^{\dagger}$ & $44.4 \pm 9.8^{\#}$ & 0.001 \\
\hline $\mathrm{TR} \mathrm{dP} / \mathrm{dt}(\mathrm{mmHg} / \mathrm{s})$ & $649.4(463.1-824.7)$ & $863.3(599.4-1,611.0)^{\wedge}$ & $468.5(381.6-804.7)^{\ddagger}$ & $<0.001$ \\
\hline TAPSE (mm) & $22.8 \pm 3.7$ & $21.9 \pm 3.8$ & $21.5 \pm 4.6$ & 0.167 \\
\hline
\end{tabular}

Values are mean \pm standard deviations, $\mathrm{n}(\%)$ or median (interquartile range). ${ }^{*}, \mathrm{P}$ value from one way ANOVA; ${ }^{\mathbf{4}}, \mathrm{P}<0.05$ compared with control; ${ }^{\dagger}, \mathrm{P}<0.01$ compared with control;, , P $<0.05$ compared with TTC; ${ }^{\ddagger}, \mathrm{P}<0.01$ compared with TTC. A, late diastolic mitral valve inflow velocity; a, sum of isovolumetric contraction time and isovolumic relaxation time; b, ejection time; $\mathrm{dP} / \mathrm{dt}$, the rate of pressure change; $\mathrm{E}$, early diastolic mitral valve inflow velocity; e', early diastolic mitral annular velocity; E/e', ratio of E to e'; FAC, fractional area change; LVEF, left ventricular ejection fraction; RVEDV, right ventricular end diastolic volume; RVEF, right ventricular ejection fraction; RVESV, right ventricular end systolic volume; RVOT, right ventricular outflow tract; TAPSE, tricuspid annular plane systolic excursion; TR, tricuspid regurgitation; TTC, tako-tsubo cardiomyopathy; VTI, velocity time integral.

patients. TTC patients fit the typical clinical profile of this syndrome: middle-aged $(65.3 \pm 13.6$ years old $)$, with a significantly high proportion of female (79.6\%), and with a similar risk for $\mathrm{CAD}$ as published, including hypertension, diabetes mellitus, hyperlipidemia and smoking. Although the heart rate and the percentage of female and hypertension were higher in TTC patients, no significant differences were found among control, TTC and CS groups in age and other risk factors for CAD. The episodes of chest pain, ST-segment elevation and abnormal $\mathrm{Q}$ wave in TTC patients were less than those in AMI/CS patients. In contrast to a mild elevation in creatine kinase $\mathrm{MB}$ /troponin T levels in TTC patients, the patients with AMI and CS had significantly higher creatine kinase $\mathrm{MB} /$ troponin $\mathrm{T}$ levels.

\section{Discrepant $L V$ and $R V$ functions/hemodynamics during TTC}

In the acute phase of TTC, LV systolic function, measured by LVEF $(38.5 \% \pm 7.4 \%$ vs. $60.2 \% \pm 7.3 \%)$ and Tei index
$(0.70 \pm 0.29$ vs. $0.37 \pm 0.18)$, significantly decreased. In contrast, RV systolic function, measured by RVEF $(58.5 \% \pm 8.2 \%$ vs. $53.5 \% \pm 5.6 \%)$, FAC $(48.1 \% \pm 9.3 \%$ vs. $42.8 \% \pm 5.1 \%)$ and $\mathrm{TR} \mathrm{dP} / \mathrm{dt}[863.3(599.4-1,611.0) v s$. $649.4(463.1-824.7) \mathrm{mmHg} / \mathrm{s}]$, was paradoxically augmented (Table 2). Both LV and RV functional/hemodynamic changes normalized after TTC recovery (Table 2).

\section{Discordant ventricular regional myocardial mechanics during TTC}

In the acute phase of TTC, the systolic longitudinal strains in 14 of $18 \mathrm{LV}$ segments were impaired (Figure 2). However, basal/middle inferior, basal inferolateral and basal lateral segments were preserved (Figure $2 A, B, C$ ). Similarly, although the strain of the apical and middle RV was impaired, the basal segment of $\mathrm{RV}$ remained functionally active (Figure $2 A, C$ ). Myocardial mechanics of both $\mathrm{LV}$ and RV exhibited an apex to base gradient (Figure 2C). Decreased myocardial deformation in both ventricles 
A
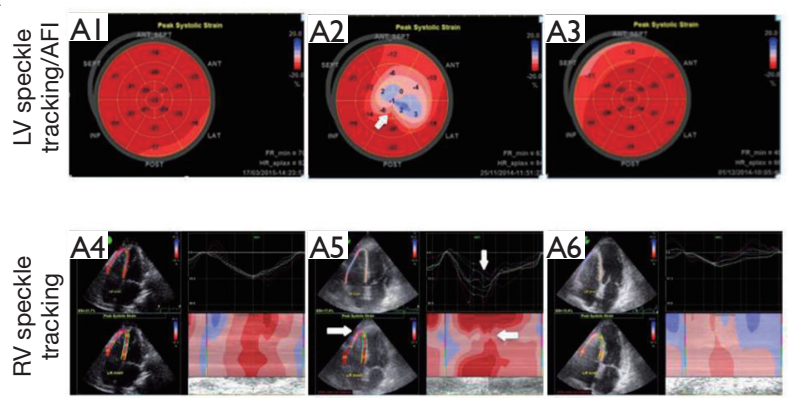

C

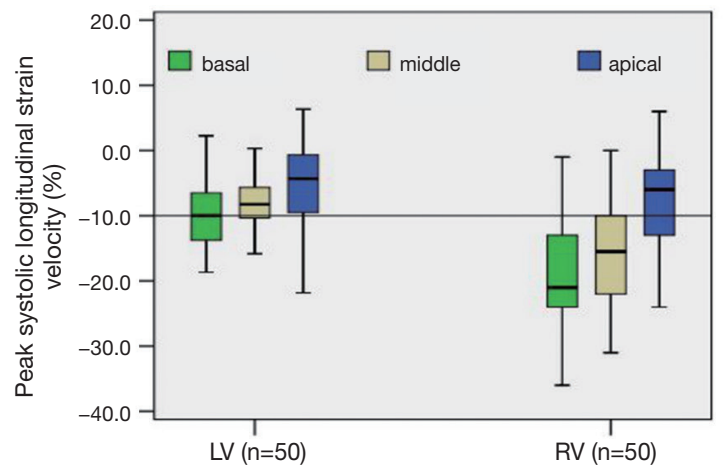

$\mathrm{B}$

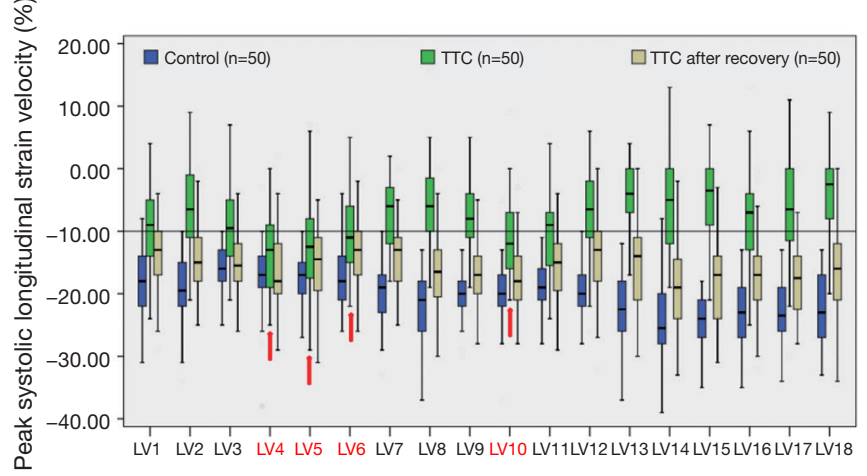

$\mathrm{D}$

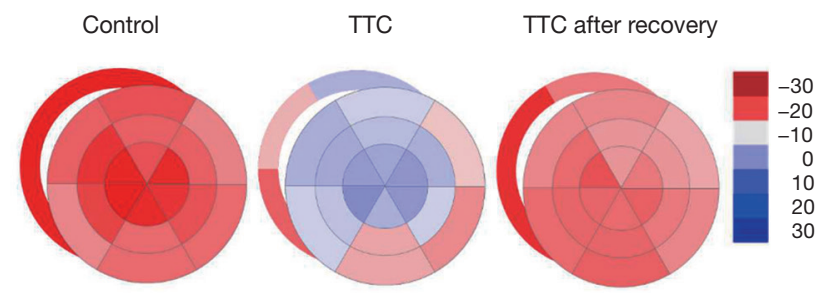

Figure 2 Changes of peak systolic longitudinal strain in LV and RV in patients with TTC. (A) The basal segments of both LV and RV remained functionally active during the acute phase of TTC. (A1,A4) Control; (A2,A5) TTC; (A3,A6) TTC after recovery. (A1-3) Speckle tracking echocardiography showed significantly decreased peak systolic longitudinal strain in most LV myocardial segments (arrow) in the automated function imaging during TTC, compared with that in control and TTC after recovery; (A4-6) speckle tracking echocardiography showed significantly decreased peak systolic longitudinal strain in the apical (arrows), but not middle and basal segments in an apical 4-chamber window during TTC, compared with that in control and TTC after recovery. The peak systolic longitudinal strain were highlighted by both digital and color codes $(18,19)$; (B) speckle tracking study showed significantly decreased peak systolic longitudinal strain in most LV myocardial segments (green), compared with those in control (blue) and TTC after recovery (yellow). However, the strain in segment 4 (basal inferior wall), 5 (basal inferolateral wall), 6 (basal anterolateral wall) and 10 (middle inferior wall) (marked as red color arrows) did not changed significantly; (C) speckle tracking study showed that the contractile function (peak systolic longitudinal strain) of LV and RV exhibit an apex to basal gradient in patients with TTC. The mean peak systolic longitudinal strain in the middle and basal segments in $\mathrm{RV}$ were higher than those in $\mathrm{LV}$; (D) a 18-segment $\mathrm{LV}$ and 3-segment RV myocardial contractile function reconstruction model showed that the basal segments of LV and RV remains functionally active during the acute phase of TTC. The peak systolic longitudinal strain was highlighted by digital color scale $(18,20)$. TTC, tako-tsubo cardiomyopathy; LV, left ventricle; RV, right ventricle.

recuperated after TTC recovery (Figure 2D).

\section{The characteristic $R V$ strain pattern remained unchanged in the patients with atypical TTC phenotype}

Fourteen of 103 (13.6\%) TTC patients had mid-cavity TTC variant [re-versed TTC (21)]. In these patients, although the $\mathrm{LV}$ apical to basal strain gradient no longer existed (Figure $3 A, B$ ), the RV apical to basal strain gradient remained unchanged, showing a comparable pattern with those patients with typical (apical) TTC phenotype (Figure 3B,C).

\section{Comparable $R V$ mechanic/functional features occurred in patients with AMI induced CS}

In 41 patients with AMI induced CS (required mechanic circulatory support devices to maintain hemodynamic 

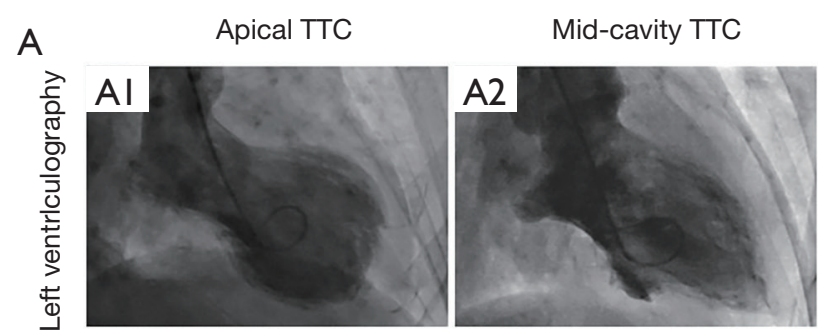

B
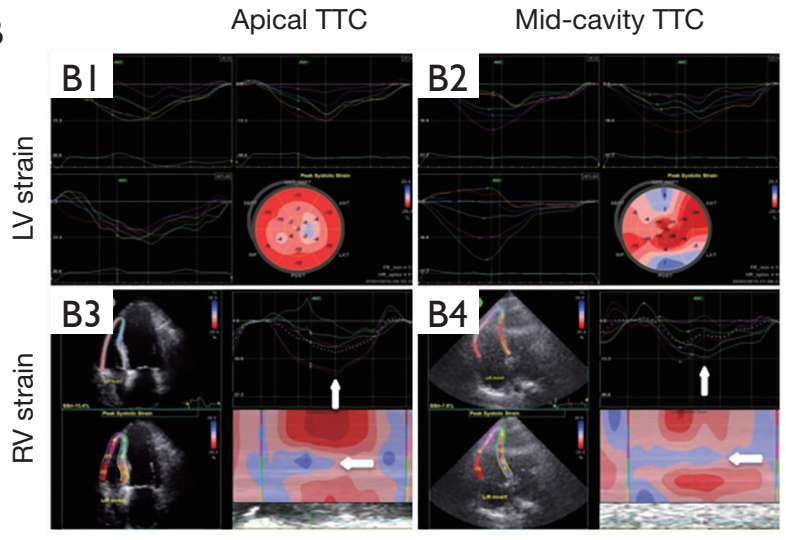

C

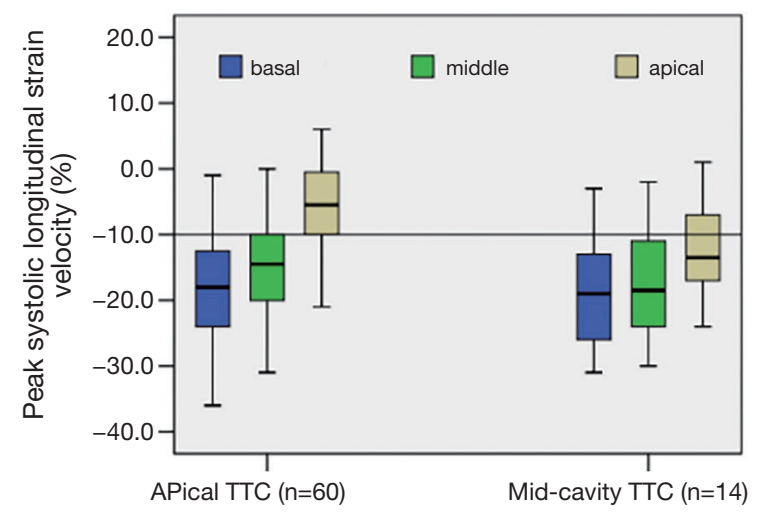

Figure 3 The RV contractile pattern remained unchanged in various TTC phenotypes. (A) Left ventriculogram depicted apical (A1) and mid-cavity (A2) TTC; (B) speckle tracking/AFI study depicted apical (B1) and mid-cavity (B2) TTC. Speckle tracking study showed similar RV contractile patterns (arrows) between apical (B3) and mid-cavity (B4) TTC; (C) there was no significant difference in $\mathrm{RV}$ contractile pattern (measured by peak systolic longitudinal strain) between apical (60 patients) and mid-cavity (14 patients, see Figure 1) TTC. TTC, tako-tsubo cardiomyopathy; $\mathrm{LV}$, left ventricle; RV, right ventricle; AFI, automated function image.

stability), LV systolic dysfunction were severely impaired (LVEF $35.9 \% \pm 10.2 \%$ and Tei index $0.69 \pm 0.36$ ). However, the RV systolic function was paradoxically enhanced [RVEF $55.5 \% \pm 6.9 \%$ and TR $\mathrm{dP} / \mathrm{dt} 812.2(537-1,085) \mathrm{mmHg} / \mathrm{s}]$ (Table 3). Meanwhile, even though their LV no longer showed any characteristic strain pattern (Figure 4A,B), $\mathrm{RV}$ constantly displayed an apical to basal strain gradient, comparable to those in TTC patients (Figure 4B,C).

\section{Discussion}

The present study characterized real time changes of myocardial mechanics and ventricular function during the acute stage of TTC. Despites comparable apical to basal strain gradients, $\mathrm{LV}$ and $\mathrm{RV}$ displayed discrepant functional/ hemodynamic status. In patients with AMI induced cardiogenic shock, RV exhibited comparable mechanic and hemodynamic characteristics.

Basic research suggested that TTC was mediated by a cardio-protective switching of epinephrine signaling transduction through the pleiotropic $\beta_{2}$-adrenergic receptors $(22,23)$. Uneven densities/sensitivities of the pleiotropic $\beta_{2}$-adrenergic receptors were found between apical and basilar myocardium (22). Clinically, advanced imaging techniques, such as cardiac positron emission tomography, have improved the tomographic visualization, and showed different metabolic status between the base and apex of the heart in TTC (24). With speckle tracking echocardiography, we previously observed a similar discordant myocardial deformation between apical and basal ventricular segments in TTC (25-27).

Consequently, the dynamic flow of blood inside RV and LV would be affected. Discordant myocardial contractile mechanics often generated a "hinge" in both ventricles during systole. Intra-ventricular circulating blood appeared to be sequestered into 2 functionally distinctive units: the "inertial" blood flow trapped inside the RV and LV apex, and the "dynamic" blood flow stirring through the base of both ventricles (Figure 5). This apparently basally dominant machinery likely facilitates more efficient intra-ventricular blood flow delivery. Since the turnover rate of the blood flow in the basal portions of the ventricles is higher than that in the apical portions of the ventricles, a substantial amount of circulatory blood would bypass apical cavities and reach left ventricular outflow tract (LVOT) through a much shorter distance. Other than being energetically efficient, this work mode avoids an abrupt increase in the LV filling pressure and irreversible damage of the vulnerable LV myocardium 
Table 3 Functional and contractile features of TTC and CS patients

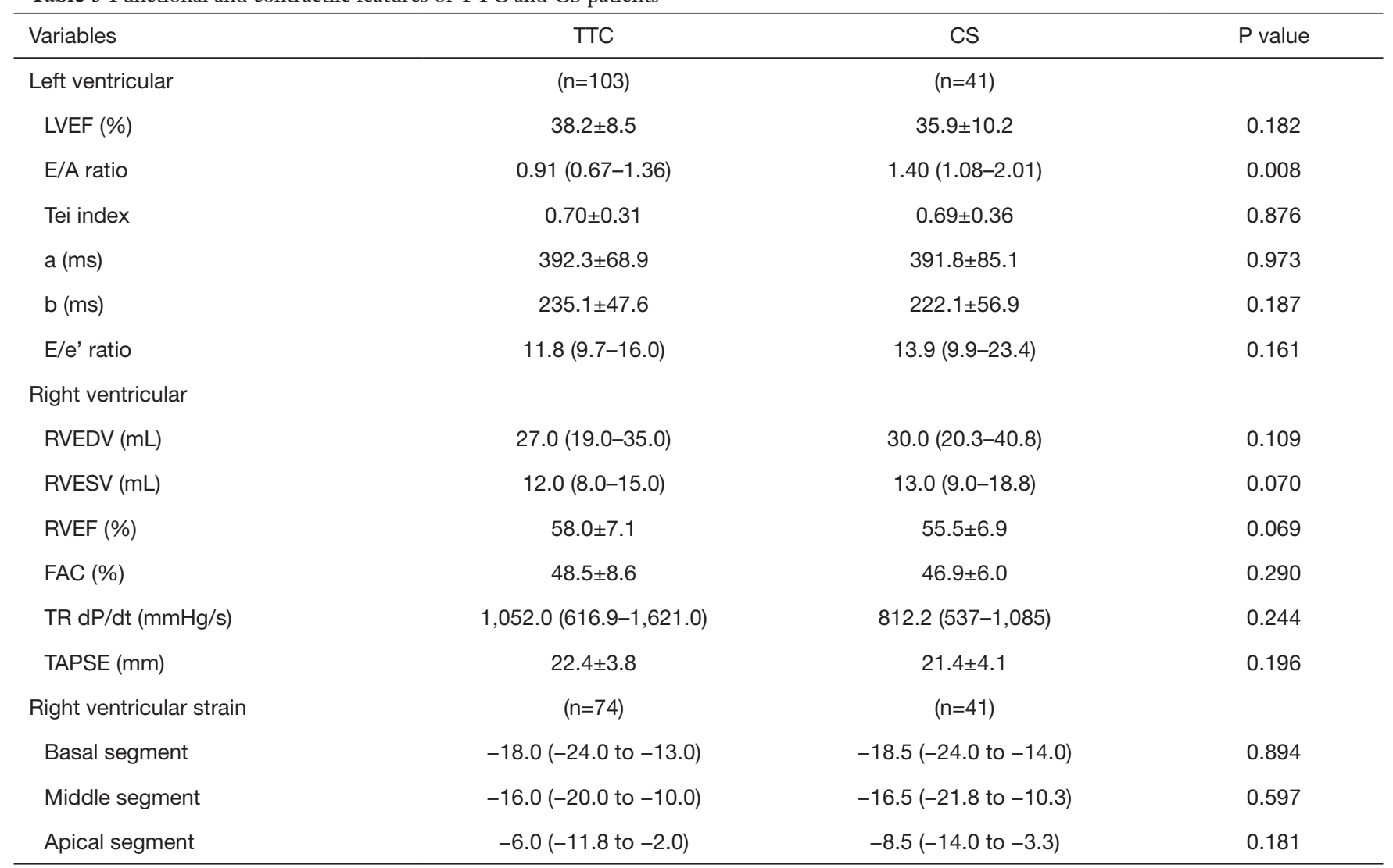

Values are mean \pm standard deviations or median (interquartile range). A, late diastolic mitral valve inflow velocity; a, sum of isovolumetric contraction time and isovolumic relaxation time; b, ejection time; $\mathrm{dP} / \mathrm{dt}$, the rate of pressure change; $\mathrm{E}$, early diastolic mitral valve inflow velocity; e', early diastolic mitral annular velocity; E/e', ratio of E to e'; FAC, fractional area change; LVEF, left ventricular ejection fraction; RVEDV, right ventricular end diastolic volume; RVEF, right ventricular ejection fraction; RVESV, right ventricular end systolic volume; RVOT, right ventricular outflow tract; TAPSE, tricuspid annular plane systolic excursion; TR, tricuspid regurgitation; TTC, tako-tsubo cardiomyopathy; CS, cardiogenic shock; VTI, velocity time integral.

$(4,25)$. When the heart is jeopardized or injured suddenly, re-adjusting the metabolism/mechanics among various myocardial regions improves its hemodynamics supporting efficacy (23-25).

The RV may play an essential role in this transitory machinery. A persistently dilated LV apex obviously restricts the dynamics of the RV apex [a unique distinguishing feature of TTC in anatomic images $(19,20,28)]$. However, due to its triangularly pyramidal shape, the basally dominant RV mechanics could maximally make up overall RV function (Table 2) and support LV preload. The characteristic RV change during TTC rapidly normalized after LV function recuperation (Figure 2D), implying that it is likely a compensatory mechanism in responses to a transient $\mathrm{LV}$ dysfunction and stroke volume reduction during TTC (through autonomic baroreflex regulation?) (21).
More evidence supports the inherent role of the RV mechanic changes in TTC: (I) although both LV and RV myocardial strain showed similar apex to base gradients in the typical TTC (apical TTC) phenotype, only the RV maintained a similar gradient (Figure 3) in the atypical TTC variant [mid-ventricular TTC (29)]; (II) the characteristic mechanical/functional changes of the RV were not confined to TTC. In patients with AMI induced CS, although LV regional contractile patterns were highly variable, both functional status and deformation characteristics of the $\mathrm{RV}$ remained constant, which were comparable to those in TTC patients (Table 3 and Figure 4) (30,31). Similar results were reported in our previous study in a sizable population of AMI patients (28).

The RV mechanic and functional features in TTC share some similarities with those in fetal hearts. During 
A
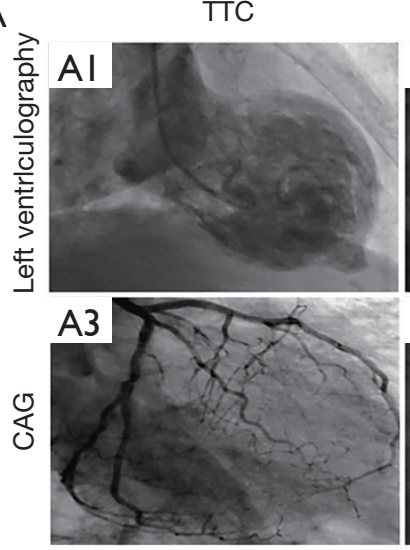

C

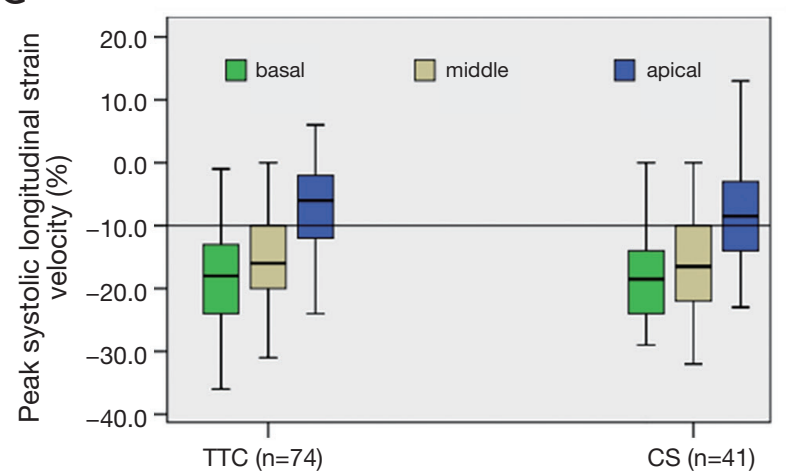

B
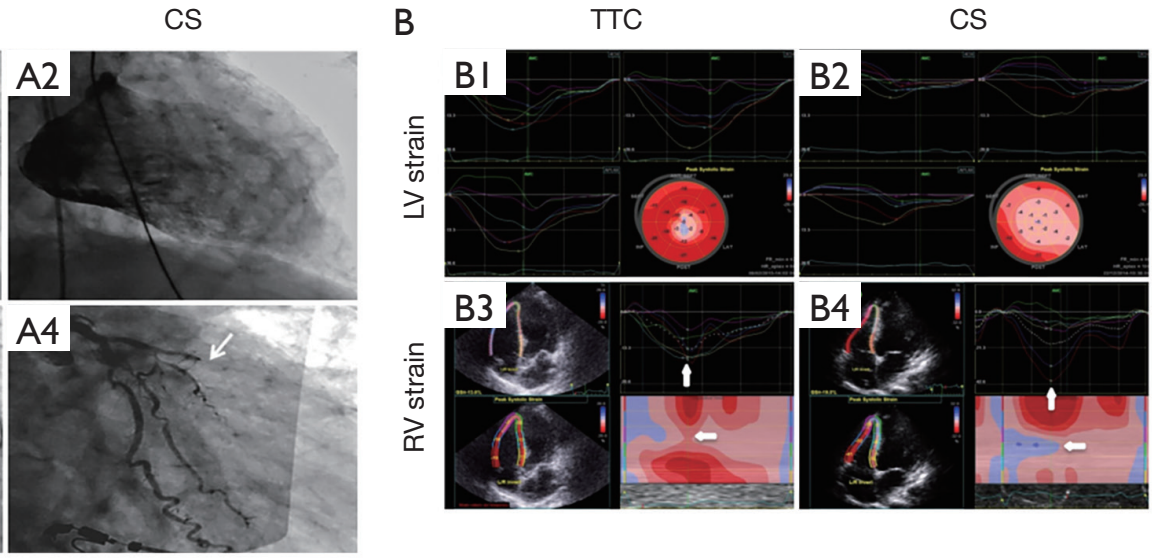

Figure 4 The RV functional and contractile features in CS secondary to AMI were similar to those in TTC. (A) Left ventriculography depicted TTC (A1) and CS/AMI (A2). CAG indicated no culprit coronary artery stenosis in TTC (A3), but a completely occluded LAD in CS/AMI (A4); (B) speckle tracking/AFI study indicated TTC (B1) and CS/AMI (B2); speckle tracking study showed similar RV contractile patterns (arrows) between TTC (B3) and CS/AMI (B4); (C) speckle tracking study showed no significant difference in RV contractile patterns (peak systolic longitudinal strain) between TTC (74 patients) and CS/AMI (41 patients, see Figure 1). TTC, tako-tsubo cardiomyopathy; CS, cardiogenic shock; AMI, acute myocardial infarction; LV, left ventricle; RV, right ventricle; CAG, coronary angiogram; $\mathrm{LAD}$, left descending artery; AFI, automated function image.

gestation, the RV is responsible for up to $70 \%$ of the entire cardiac output (through a patent foramen ovale and patent ductus arteriosus). Fetal RV demonstrated significantly more active functional status than the $\mathrm{LV}$, and both $\mathrm{LV}$ and RV exhibited an apical to basal strain gradient (32-34). Through the transition from fetal to neonatal life (LV function becomes dominant), above mechanic/ functional features disappear rapidly (32). Do adult hearts still retain the ability (memory imprint) to re-activate this primitive "RV dominant" work model as an atavistically salvage mechanism during TTC?

These peculiar alterations in the myocardial mechanics appear to preserve hemodynamic stability and benign clinical phenotypes during most TTC episodes $(3,4)$.
Interruptions to this possible "salvage mechanism" can actually trigger a transition from a physiological to a pathophysiological process. In patients with pre-existing isolated basal septal hypertrophy (IBSH), volume depletion and over-hypercontractility of ventricular base can precipitate dynamic LVOT obstruction (35), and aggravate mitral regurgitation (MR) (36). A "volume maldistribution condition" $(3,4)$ may result in simultaneous hypotension and pulmonary edema. Decreased cardiac output could further magnify secondary sympathetic output and aggravate inotropic and chronotropic effects of the heart, and generate a vicious hemodynamic cycle $(4,37)$. Therefore, maintaining sufficient $L V$ preload and increasing peripheral vascular pressure/tension (instead of catecholamine pressors) are 


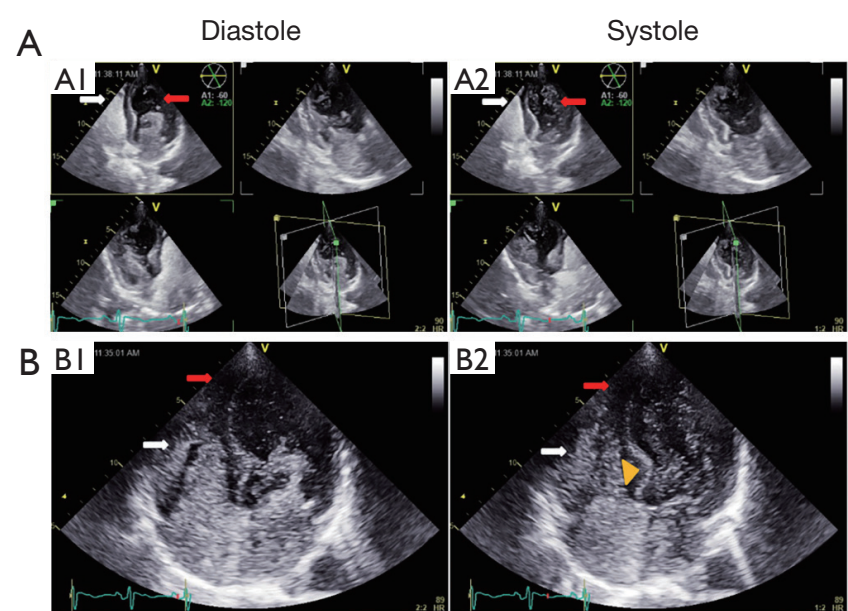

Figure 5 A right and basal dominant distribution of echocardiographic contrast during the acute phase of TTC. (A) A 2-dimensional (3-axis) echocardiogram in a TTC patient showed real-time diastolic (A1) and systolic (A2) contrast distribution in apical 4-, 2- and 3-chamber windows. There was a significant discrepancy in the density of contrast between RV (white arrows) and LV (red arrows); (B) the ventricular contrast distribution exhibited an apex (red arrows) to base (white arrows) gradient in both diastole (B1) and systole (B2). Yellow arrow head: the "hinge" section of left ventricle during the systole in TTC. TTC, takotsubo cardiomyopathy; LV, left ventricle; $\mathrm{RV}$, right ventricle.

likely the most efficient strategies to avoid adverse events in such circumstances (38-40). While the actual effect of intra-aortic balloon pump is uncertain, whether baroreflex activation therapy (41), or percutaneous Impella ventricular assist device (42), can better support hemodynamics in the malignant TTC phenotypes would be the research focus down the road.

\section{Limitations}

Our study has several limitations: (I) TTC is a highly dynamic and time-variant process. Clinically, the timing to apply real-time cardiac imaging, including echocardiography or cardiac catheterization, is often affected by multiple factors, hence is variable. Accordingly, the measurement of both contractile function and hemodynamics might vary at different time-points, and introduces bias into the study; (II) we have included the initial clinical and laboratory data including comprehensive echocardiographic studies in all of the consecutive TTC patients diagnosed in our institute over 8 years. Our institution serves as the major referral center for patients in central New York area, and some of the follow-up echocardiographic studies were actually performed elsewhere in our network, which were not suitable for post-process with strain analysis. The lack of availability of follow-up echocardiographic data in some patients limited our comparison during and after TTC; (III) the accurate assessment and measurement of the RV structure and function/hemodynamics with routine echocardiographic techniques was sometimes challenging, due to the complex RV anatomy, and poor visualization of RV apex. Although the application of newly developed contrast echocardiography (43) could possibly address this problem, the retrospective nature of this study limited the choices we had with respect to methodology.

\section{Acknowledgements}

None.

\section{Footnote}

Conflicts of Interest: The authors have no conflicts of interest to declare.

Ethical Statement: The study was approved by the institutional research board of the Human Subjects Committee of SUNY Upstate Medical University.

\section{References}

1. Pelliccia F, Parodi G, Greco C, et al. Comorbidities frequency in Takotsubo syndrome: an international collaborative systematic review including 1109 patients. Am J Med 2015;128:654.e11-9.

2. Templin C, Ghadri JR, Diekmann J, et al. Clinical Features and Outcomes of Takotsubo (Stress) cardiomyopathy. N Engl J Med 2015;373:929-38.

3. Citro R, Rigo F, D'Andrea A, et al. Echocardiographic correlates of acute heart failure, cardiogenic shock, and inhospital mortality in tako-tsubo cardiomyopathy. JACC Cardiovasc Imaging 2014;7:119-29.

4. Liu K, Krone RJ. What truly causes the adverse outcome in Tako-Tsubo cardiomyopathy? JACC Cardiovasc Imaging 2014;7:742-3.

5. Prasad A, Lerman A, Rihal CS. Apical ballooning syndrome (Tako-Tsubo or stress cardiomyopathy): a mimic of acute myocardial infarction. Am Heart J 2008;155:408-17. 
6. Scantlebury DC, Prasad A. Diagnosis of Takotsubo cardiomyopathy. Circ J 2014;78:2129-39.

7. Lang RM, Badano LP, Mor-Avi V, et al. Recommendations for cardiac chamber quantification by echocardiography in adults: an update from the American Society of Echocardiography and the European Association of Cardiovascular Imaging. Eur Heart J Cardiovasc Imaging 2015;16:233-70.

8. Rudski LG, Lai WW, Afilalo J, et al. Guidelines for the echocardiographic assessment of the right heart in adults: a report from the American Society of Echocardiography endorsed by the European Association of Echocardiography, a registered branch of the European Society of Cardiology, and the Canadian Society of Echocardiography. J Am Soc Echocardiogr 2010;23:685-713.

9. Wacholder S, McLaughlin JK, Silverman DT, et al. Selection of controls in case - control studies. I. Principles. Am J Epidemiol 1992;135:1019-28.

10. Szymanski FM, Filipiak KJ. Cardiogenic shock - diagnostic and therapeutic options in the light of new scientific data. Anaesthesiol Intensive Ther 2014;46:301-6.

11. Nishimura RA, Carabello BA. Hemodynamics in the cardiac catheterization laboratory of the 21 st century. Circulation 2012;125:2138-50.

12. Lei J, Dhamoon AS, Wang J, et al. Walking the tightrope: Using quantitative Doppler echocardiography to optimize ventricular filling pressures in patients hospitalized for acute heart failure. Eur Heart J Acute Cardiovasc Care 2016;5:130-40.

13. Voelkel NF, Quaife RA, Leinwand LA, et al. Right ventricular function and failure: report of a National Heart, Lung, and Blood Institute working group on cellular and molecular mechanisms of right heart failure. Circulation 2006;114:1883-91.

14. Hung CL, Verma A, Uno H, et al. Longitudinal and circumferential strain rate, left ventricular remodeling, and prognosis after myocardial infarction. J Am Coll Cardiol 2010;56:1812-22.

15. Liu K, Krone R. Evaluation of coronary steal in myocardium supplied by coronary collaterals: the role of speckle tracking analysis in resting and stress echocardiography. Echocardiography 2013;30:1111-7.

16. Lemarié J, Huttin O, Girerd N, et al. Usefulness of Speckle-Tracking Imaging for Right Ventricular Assessment after Acute Myocardial Infarction: A Magnetic Resonance Imaging/Echocardiographic Comparison within the Relation between Aldosterone and Cardiac Remodeling after Myocardial Infarction Study. J Am Soc
Echocardiogr 2015;28:818-27.e4.

17. Fukuda Y, Tanaka H, Sugiyama D, et al. Utility of right ventricular free wall speckle-tracking strain for evaluation of right ventricular performance in patients with pulmonary hypertension. J Am Soc Echocardiogr 2011;24:1101-8.

18. Oxborough D, Shave R, Warburton D, et al. Dilatation and dysfunction of the right ventricle immediately after ultraendurance exercise: exploratory insights from conventional two-dimensional and speckle tracking echocardiography. Circ Cardiovasc Imaging 2011;4:253-63.

19. McConnell MV, Solomon SD, Rayan ME, et al. Regional right ventricular dysfunction detected by echocardiography in acute pulmonary embolism. Am J Cardiol 1996;78:469-73.

20. Liu K, Carhart R. "Reverse McConnell's sign?": a unique right ventricular feature of Takotsubo cardiomyopathy. Am J Cardiol 2013;111:1232-5.

21. Ali A, Redfors B, Omerovic E. How baroreceptor dysfunction could predispose to the takotsubo syndrome. Int J Cardiol 2015;182:105-6.

22. Lyon AR, Rees PS, Prasad S, et al. Stress (Takotsubo) cardiomyopathy - a novel pathophysiological hypothesis to explain catecholamine-induced acute myocardial stunning. Nat Clin Pract Cardiovasc Med 2008;5:22-9.

23. Paur H, Wright PT, Sikkel MB, et al. High levels of circulating epinephrine trigger apical cardiodepression in a beta2-adrenergic receptor/Gi-dependent manner: a new model of Takotsubo cardiomyopathy. Circulation 2012;126:697-706.

24. Christensen TE, Ahtarovski KA, Bang LE, et al. Basal hyperaemia is the primary abnormality of perfusion in Takotsubo cardiomyopathy: a quantitative cardiac perfusion positron emission tomography study. Eur Heart J Cardiovasc Imaging 2015;16:1162-9.

25. Sun Z, Singh P, Wei TM, et al. Is the heart really stressed out of energy? JACC Cardiovasc Imaging 2016;9:633-5.

26. Liu K. Letter by Liu regarding article, "systolic and diastolic mechanics in stress cardiomyopathy". Circulation 2015;131:e371.

27. Liu K. Does the heart have a mind of its own? Prevent adverse outcome from Takotsubo cardiomyopathy. Heart 2014;100:1816.

28. Singh A, Lei J, Kozman H, et al. "Reverse McConnell's Sign": A unique right ventricular mechanical and functional feature in Tako-Tsubo cardiomyopathy. Circulation 2014;130:A13894.

29. Hurst RT, Prasad A, Askew JW 3rd, et al. Takotsubo 
cardiomyopathy: a unique cardiomyopathy with variable ventricular morphology. JACC Cardiovasc Imaging 2010;3:641-9.

30. Hanna M, Finkelhor RS, Shaw WF, et al. Extent of right and left ventricular focal wall-motion abnormalities in differentiating transient apical ballooning syndrome from apical dysfunction as a result of coronary artery disease. J Am Soc Echocardiogr 2007;20:144-50.

31. Park SM, Prasad A, Rihal C, et al. Left ventricular systolic and diastolic function in patients with apical ballooning syndrome compared with patients with acute anterior ST-segment elevation myocardial infarction: a functional paradox. Mayo Clin Proc 2009;84:514-21.

32. Schubert U, Muller M, Norman M, et al. Transition from fetal to neonatal life: changes in cardiac function assessed by speckle-tracking echocardiography. Early Hum Dev 2013;89:803-8.

33. Kim SH, Miyakoshi K, Kadohira I, et al. Comparison of the right and left ventricular performance during the fetal development using velocity vector imaging. Early Hum Dev 2013;89:675-81.

34. Willruth AM, Geipel AK, Berg CT, et al. Comparison of global and regional right and left ventricular longitudinal peak systolic strain, strain rate and velocity in healthy fetuses using a novel feature tracking technique. J Perinat Med 2011;39:549-56.

35. Ranasinghe I, Ayoub C, Cheruvu C, et al. Isolated hypertrophy of the basal ventricular septum: characteristics of patients with and without outflow tract obstruction. Int J Cardiol 2014;173:487-93.

36. Izumo M, Nalawadi S, Shiota M, et al. Mechanisms of

Cite this article as: Lei J, Sun Z, Lyu L, Green RG, Scalzetti E, Feiglin D, Wang J, Liu K. Mechanical interventricular dependency supports hemodynamics in tako-tsubo cardiomyopathy. J Thorac Dis 2018;10(5):3027-3038. doi: 10.21037/jtd.2018.04.143 acute mitral regurgitation in patients with takotsubo cardiomyopathy: an echocardiographic study. Circ Cardiovasc Imaging 2011;4:392-8.

37. Boppana VS, Bhatta L, Liu K. Reversible TR in acute and chronic cardiomyopathy: medical versus surgical management? JACC Cardiovasc Imaging 2013;6:920-1.

38. Liu K, Sun Z, Wei T. "Reverse McConnell's Sign": Interpreting Interventricular Hemodynamic Dependency and Guiding the Management of Acute Heart Failure during Takotsubo Cardiomyopathy. Clin Med Insights Cardiol 2015;9:33-40.

39. Citro R, Lyon AR, Meimoun P, et al. Standard and advanced echocardiography in takotsubo (stress) cardiomyopathy: clinical and prognostic implications. J Am Soc Echocardiogr 2015;28:57-74.

40. Medeiros K, O'Connor MJ, Baicu CF, et al. Systolic and diastolic mechanics in stress cardiomyopathy. Circulation 2014;129:1659-67.

41. Abraham WT, Zile MR, Weaver FA, et al. Baroreflex Activation Therapy for the Treatment of Heart Failure With a Reduced Ejection Fraction. JACC Heart Fail 2015;3:487-96.

42. Rashed A, Won S, Saad M, et al. Use of the Impella 2.5 left ventricular assist device in a patient with cardiogenic shock secondary to takotsubo cardiomyopathy. BMJ Case Rep 2015. doi: 10.1136/bcr-2014-208354.

43. Cheng Y, Villarreal D, Liu K. Pulmonary arterial hypertension versus pulmonary venous hypertension: how much information can contrast Doppler echocardiography provide? Echocardiography 2013;30:733-5. 